\title{
Prévoir le risque de fermeture de l'angle tel que défini par la classification de Shaffer à l'aide de la tomographie par cohérence optique du segment antérieur : Une approche simple
}

\author{
Dan Samaha O.D., M. Sc \\ Université de Montréal, \\ École d'optométrie
}

Sébastien Gagné, M.D., FRSC Institut de l'œil des Laurentides

Marie-Eve Corbeil, O.D., M. Sc Université de Montréal, École d'optométrie

Pierre Forcier, O.D., M. Sc Université de Montréal, École d'optométrie
OBJECTIF

Proposer une méthode non invasive simple pour le dépistage des patients à risque de fermeture de l'angle en utilisant l'imagerie du segment antérieur à l'aide de la TCO.

\section{MÉTHODES}

Des examens radiologiques des angles irido-cornéens en nasal et en temporal ont été réalisés au moyen de la TCO chez des patients référés pour suspicion de glaucome. Après l'identification de la ligne de Schwalbe, une droite fut tracée jusqu'au point de l'iris le plus rapproché (S-I) à l'aide de l'outil de compas intégré de l'appareil. Une gonioscopie a été effectuée et la méthode de Shaffer a été utilisée pour grader l'ouverture de l'angle afin d'évaluer la corrélation entre les deux méthodes.

\section{RÉSULTATS}

Trente-quatre images étaient disponibles pour analyse. Les coefficients de corrélation de Spearman entre les mesures S-I et les grades de Shaffer se sont établis à 0,81 et 0,77 pour les quadrants nasal et temporal respectivement. Les calculs intra-observateur des coefficients de corrélation intraclasse avaient une excellente reproductibilité ( 0,98 et 0,99 pour les angles en nasal et en temporal) et on a observé une excellente corrélation inter-observateur $(0,94$ et 0,93$)$. La valeur diagnostique $S-I$ pour les angles à risque de fermeture a été provisoirement établie à $330 \mathrm{~mm}$.

\section{CONCLUSION}

La mesure S-I corrèle fortement avec les résultats de la gonioscopie et pourrait être une solution de rechange appropriée pour évaluer le risque de fermeture de l'angle.

\section{MOTS CLÉS :}

imagerie de l'angle de la chambre antérieure, TCO-SA, glaucome, iridotomie périphérique au laser, tomographie par cohérence optique

À l'heure actuelle, environ 15 millions de personnes dans le monde souffrent de glaucome primitif à angle fermé $(\mathrm{GPAF})^{1}$; cette maladie constitue la première cause de cécité irréversible médicalement ou chirurgicalement. Ce type de glaucome est causé par l'accolement de la partie périphérique de l'iris sur le réseau trabéculaire, ce qui empêche l'écoulement de l'humeur aqueuse par l'angle de la chambre antérieure. Le bloc pupillaire, qui est à l'origine de $90 \%$ des cas de $\mathrm{GPAF}^{2}$, est causé par un accolement de l'iris et de la face antérieure du cristallin, qui limite l'écoulement de l'humeur aqueuse de la chambre postérieure vers la chambre antérieure. L'accumulation de l'humeur aqueuse dans la chambre postérieure pousse l'iris de façon périphérique vers l'avant (iris bombé) et, éventuellement, cause la fermeture de l'angle. Ce mécanisme peut se produire de façon aiguë, intermittente ou chronique. La prévalence moyenne du GPAF dans le monde est actuellement estimée à $0,69 \%$. 
Au cours des 40 dernières années, de nombreux facteurs de risque ont été identifiés, comme l'hypermétropie, un faible diamètre cornéen et l'insertion élevée de l'iris, mais l'observation de l'angle irido-cornéen par gonioscopie demeure la norme clinique pour le dépistage de cette maladie. Bien qu'elle soit facile à réaliser, la gonioscopie présente ses limites, à savoir la nécessité d'un contact direct avec une cornée anesthésiée, les résultats faussés en raison de la pression sur la cornée ou d'une trop grande quantité de lumière ambiante qui provoque une ouverture artificielle de l'angle. De plus, de nombreuses études ont démontré la grande variabilité des résultats obtenus par gonioscopie chez les observateurs expérimentés ${ }^{3-5}$.

L'utilisation de la tomographie par cohérence optique (TCO) en ophtalmologie était auparavant réservée à l'étude du segment postérieur (rétine) ${ }^{6}$. De nos jours, il est possible d'acquérir rapidement des images en haute définition du segment antérieur, tout en éliminant le besoin de contact avec la cornée?

De nombreuses études ont déjà décrit différentes approches pour définir quantitativement l'ouverture de l'angle irido-cornéen ${ }^{7-10}$ en utilisant l'éperon scléral comme point de repère anatomique. Comme il n'est pas identifiable dans 20 à $30 \%$ des cas $^{8}$, les auteurs se sont rapidement tournés vers la ligne de Schwalbe comme nouveau point de repère anatomique ${ }^{9-10}$, puisqu'elle peut être visualisée et identifiée sur plus de $95 \%$ des images du segment antérieur acquises par TCO. Les modèles actuels de mesure calculent les caractéristiques des segments antérieurs, comme la distance d'ouverture de l'angle (AOD), la surface de l'iris trabéculaire (TISA) et la distance d'ouverture de l'angle de la ligne de Schwalbe (SL-AOD) dont la fiabilité inter-observateur et intra-observateur a été démontrée. Toutefois, ces mesures reposent toujours sur des manipulations de compas par les observateurs à plusieurs points d'image et peuvent faire l'objet d'erreurs de manipulation. Bien que certains instruments de TCO puissent facilement calculer ces mesures, beaucoup d'autres dispositifs ne le peuvent pas. Il est donc nécessaire d'exporter les images sur une autre plateforme, ce qui augmente le temps nécessaire à l’analyse des images.

Dans cette étude, nous proposons une approche simple utilisant la tomographie par cohérence optique du segment antérieur, ou TCO-SA, qui permettrait d'obtenir des images et de quantifier l'ouverture de l'angle irido-cornéen rapidement en déterminant la distance minimale entre la ligne de Schwalbe et l'iris. Il pourrait être plus simple de tracer une seule ligne de l'iris à la ligne de Schwalbe que d'exporter une image et d'utiliser un outil de compas à plusieurs points de l'angle du segment antérieur. Cet outil pourrait être utilisé comme méthode de dépistage plus rapide et non invasive pour les patients chez qui on soupçonne la fermeture de l'angle.

\section{MÉTHODES}

Participants

Dans cette étude prospective, 40 sujets blancs ( 22 femmes, 18 hommes) ont été recrutés à la clinique de glaucome de l'Institut de l'Eil des Laurentides (IOL) située à Boisbriand (Québec). Les patients ont été vus pour la première fois à l'Institut et avaient été référés par leur optométriste respectif pour suspicion de glaucome, en raison, notamment, d'une pression intraoculaire élevée ou d'un rapport cupule-disque suspect., L'étude a reçu l'approbation du comité d'éthique de l'Université de Montréal (certificat \#13-029-CERES-D) et le consentement éclairé par écrit de tous les participants a été obtenu, conformément aux principes de la Déclaration d'Helsinki.

Tous les sujets ont subi un examen ophtalmique comprenant l'acuité visuelle, la biomiscroscopie par lampe à fente, la tonométrie par applanation (tonomètre de Goldmann) et la gonioscopie en chambre noire. Les sujets ayant des antécédents de chirurgie intraoculaire, de traumatisme pénétrant, de syndrome de dispersion des pigments ou toute opacité ou anomalie cornéenne en raison de laquelle il n'était pas possible de réaliser une TCO-SA ont été exclus. Les personnes ayant déjà subi une iridotomie périphérique au laser n’ont pas été exclues.

Un spécialiste du glaucome expérimenté (SG) a effectué tous les examens susmentionnés et a utilisé un gonioscope à 4 miroirs G4 Volk dans une salle d'examen sombre. À l'aide d'un faisceau à fente de $1 \mathrm{~mm}$ de largeur et de $7 \mathrm{~mm}$ de hauteur, en décalant verticalement la lumière pour permettre la visualisation de l'angle cornéen et éviter l'exposition à la lumière par la pupille, on a gradé l'angle dans les quatre quadrants (supérieur, inférieur, nasal et temporal) des deux yeux en utilisant la classification de Shaffer. Selon l'American Academy of Ophthalmology (AAO), cette classification est la plus couramment utilisée. La classification de Shaffer est la suivante : niveau 4 pour un angle ouvert (35-45 degrés); niveau 3 pour un angle ouvert (20-35 degrés); niveau 2 pour indiquer un angle avec un risque de fermeture (20 degrés); niveau 1 ou moins pour un quadrant (10 degrés ou moins) pour indiquer un risque élevé de fermeture. On a procédé à une gonioscopie par indentation pour vérifier la présence ou l'absence de synéchie antérieure périphérique (SAP). Les patients présentant des signes de SAP ont été exclus. 


\section{IMAGERIE DE LA CHAMBRE ANTÉRIEURE ET DE L'ANGLE}

\section{TCO-SA}

L'imagerie de l'angle a été réalisée sur des pupilles non dilatées, avec un système de tomographie par cohérence optique en domaine spectral (TCO dans le domaine de Fourier) ou TCO-DS (Spectralis, Heidelberg Engineering, Allemagne). L'appareil utilise une source de lumière d'une longueur d'onde de $820 \mathrm{~nm}$ et a une vitesse de balayage de 40000 A-scans par seconde. La résolution latérale de l'image peut atteindre 7 microns par pixel. Un objectif pour segment antérieur a été utilisé pour l'imagerie du segment antérieur. Tous les balayages ont été effectués sous un éclairage uniforme de 3,5 lux, mesuré à l'aide d'un luxmètre (modèle Sekonic L-308DC, DigiCineMate, North White Plains, NY). Des balayages ont été effectués au moyen du module « angle-angle » pour visualiser les angles en nasal et en temporal simultanément (figure 1) sur la même image. Une cible de fixation a été utilisée pour guider correctement le regard du sujet vers sa position principale. Seules des images de quadrants nasal et temporal ont été acquises, en raison de difficultés techniques dans l'acquisition par balayage des quadrants supérieur et inférieur.

Figure 1 : Imagerie du segment antérieur à l'aide de la tomographie par cohérence optique Spectralis; angles nasal et temporal en une seule capture.

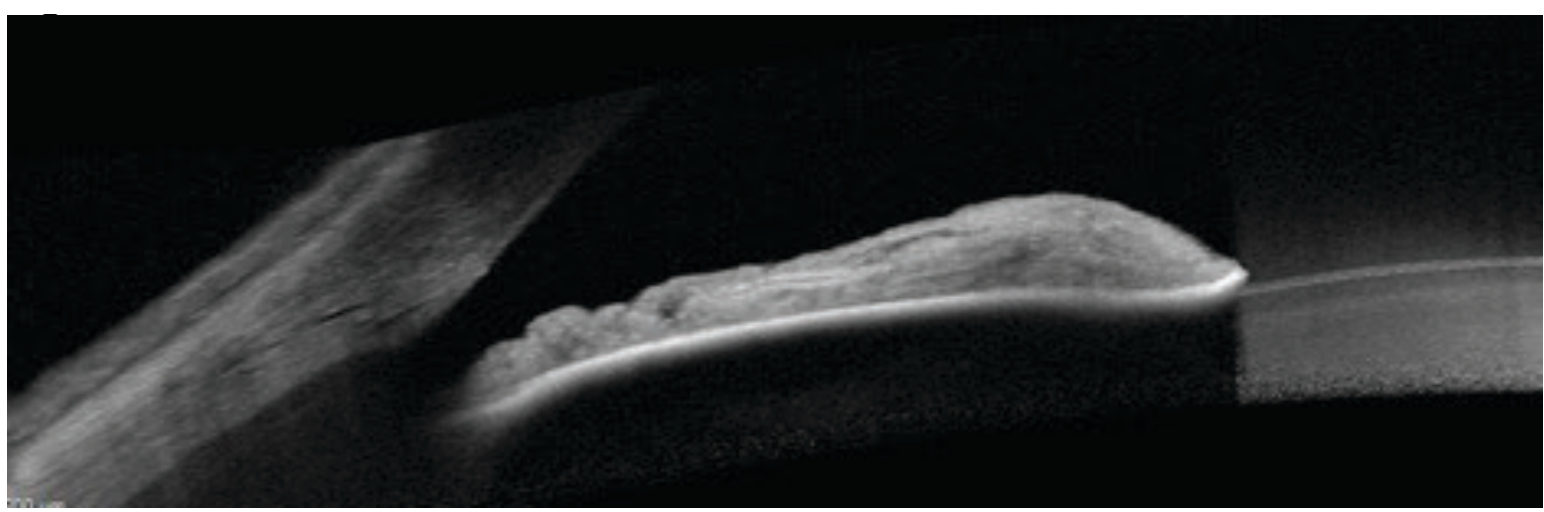

\section{Évaluation quantitative : S-I}

Les images présentant des artefacts qui entravaient l'identification du point de repère anatomique (ligne de Schwalbe) ont été exclues. Deux observateurs (DS, SG) ont effectué les mesures de façon indépendante. Après avoir identifié la ligne de Schwalbe, une ligne a été tracée entre celle-ci et le point le plus proche de l'iris à l'aide de l'outil intégré de traceur de ligne du Spectralis. La mesure était instantanément affichée en microns et l'observateur pouvait donc facilement identifier la plus petite mesure possible. Nous avons appelé cette mesure S-I (figure 2). L'observateur DS a réévalué les images réorganisées de façon aléatoire deux semaines plus tard.

Figure 2 : Angle de la chambre antérieure dans une image par tomographie par cohérence optique du segment antérieur. (A) On peut clairement identifier la ligne de Schwalbe comme la petite indentation signifiant la fin de l'endothélium cornéen. (B) Mesure S-I à l'aide de l'outil de mesure intégré. La distance est affichée automatiquement et indiquée ici comme étant 311 um.

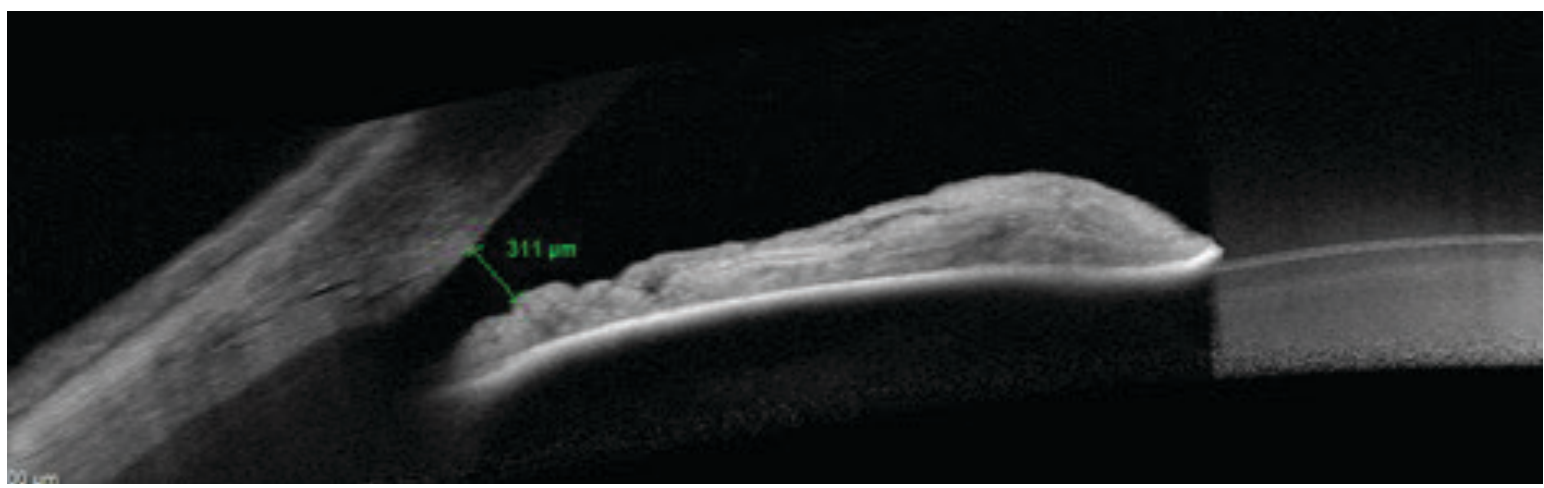




\section{Analyse statistique}

Pour chaque sujet, un œil a été sélectionné au hasard pour l'analyse des données. Les analyses statistiques ont été effectuées à l'aide de la version 25 de SPSS (élaborée par SPSS, Chicago, IL). Le test du chi carré a été utilisé pour comparer des données catégorielles. Pour chaque grade par gonioscopie, les tests ANOVA et Tukey Kramer ont été utilisés pour analyser les paires de moyenne de la valeur S-I et évaluer si la différence était significative, la mesure S-I étant spécifique au quadrant (nasal et temporal). Une analyse de corrélation des coefficients intraclasse (CCI) a été utilisée pour évaluer la reproductibilité inter-observateur et intra-observateur. Finalement, le coefficient rhô de Spearman a été utilisé pour évaluer la force de l'association entre la valeur S-I et le classement par gonioscopie.

\section{RÉSULTATS}

Tous les patients étaient de race blanche. L'âge moyen (écart type ET) était de 59,7 ans (7,67, plage 41-75) et l'équivalent sphérique moyen était de +0,75 1,50 D. Huit images ont été exclues en raison de leur piètre qualité, ce qui a laissé 32 images à analyser (14 yeux de droite et 18 yeux de gauche). Il n'y avait pas de différences significatives entre l'âge moyen et le sexe pour tous les sujets inclus (analyse chi carré, données non présentées). Dix-sept patients présentaient des angles à risque de fermeture par gonioscopie (grade Shaffer 1 ou moins), dont cinq qui avaient déjà subi une iridotomie périphérique au laser. La corrélation entre les mesures S-I et le classement par gonioscopie a été jugée statistiquement significative (Spearman $\mathrm{r}=0,81$ et 0,79 pour les quadrants nasal et temporal respectivement, $\mathrm{p}<0,05)$.

Le tableau 1 présente les résultats du test DSH de Tukey Kramer et illustre les différences entre les moyennes S-I pour chaque grade gonioscopique pour les quadrants nasal et temporal, respectivement. Les valeurs positives indiquent une paire d'angle dont la valeur diffère de façon statistiquement significative. Par exemple, en regardant le tableau 1, la première ligne compare la valeur moyenne S-I pour un grade 4 de Shaffer avec la valeur moyenne S-I pour tous les autres grades de Shaffer. On peut observer que la valeur moyenne S-I pour un grade 4 est très différente de la valeur S-I pour un grade 2, 1 ou 0 de Shaffer. La figure 3 illustre graphiquement ces résultats, accompagnés des cercles de comparaison.

Tableau 1 : Comparaison entre les moyennes à l'aide du test DSH de Tukey-Kramer (valeur S-I obtenue par TCO par rapport au grade défini par gonioscopie)

\begin{tabular}{|c|c|c|c|c|c|}
\hline Grade SHAFFER & 4 & 3 & 2 & 1 & 0 \\
\hline 4 & $\mathrm{X}$ & $-14,1978$ & 28,342 & 126,3621 & 106,3621 \\
\hline 3 & $-14,1978$ & $\mathrm{x}$ & 5,988 & 19,90661 & 1,64431 \\
\hline 2 & 28,3242 & 5,988 & $\mathrm{x}$ & $-3,427$ & $-9,621$ \\
\hline 1 & 126,8587 & 19,90661 & $-3,427$ & $\mathrm{x}$ & $-103,715$ \\
\hline 0 & 106,3621 & 1,64431 & $-9,621$ & $-103,715$ & $\mathrm{x}$ \\
\hline
\end{tabular}

*Les valeurs positives révèlent des paires de moyennes qui sont statistiquement différentes

\begin{tabular}{|ccc|}
\hline GRADE & & MOYENNE \\
\hline 4 & A & 451,00 \\
\hline 3 & A B & 354,60 \\
\hline 2 & B C & 301,71 \\
\hline 1 & C & 232,81 \\
\hline 0 & C & 226,25 \\
\hline
\end{tabular}

Les niveaux qui ne sont pas reliés par la même lettre présentent une différence importante 
Figure 3 : Comparaison des moyennes de la mesure S-I en nasal par TCO avec le classement par gonioscopie à l'aide de l'analyse DSH de Tukey-Kramer. L'indice Younen a été utilisé pour déterminer la valeur seuil S-I entre les angles ouverts et les angles à risque de fermeture (ligne rouge).

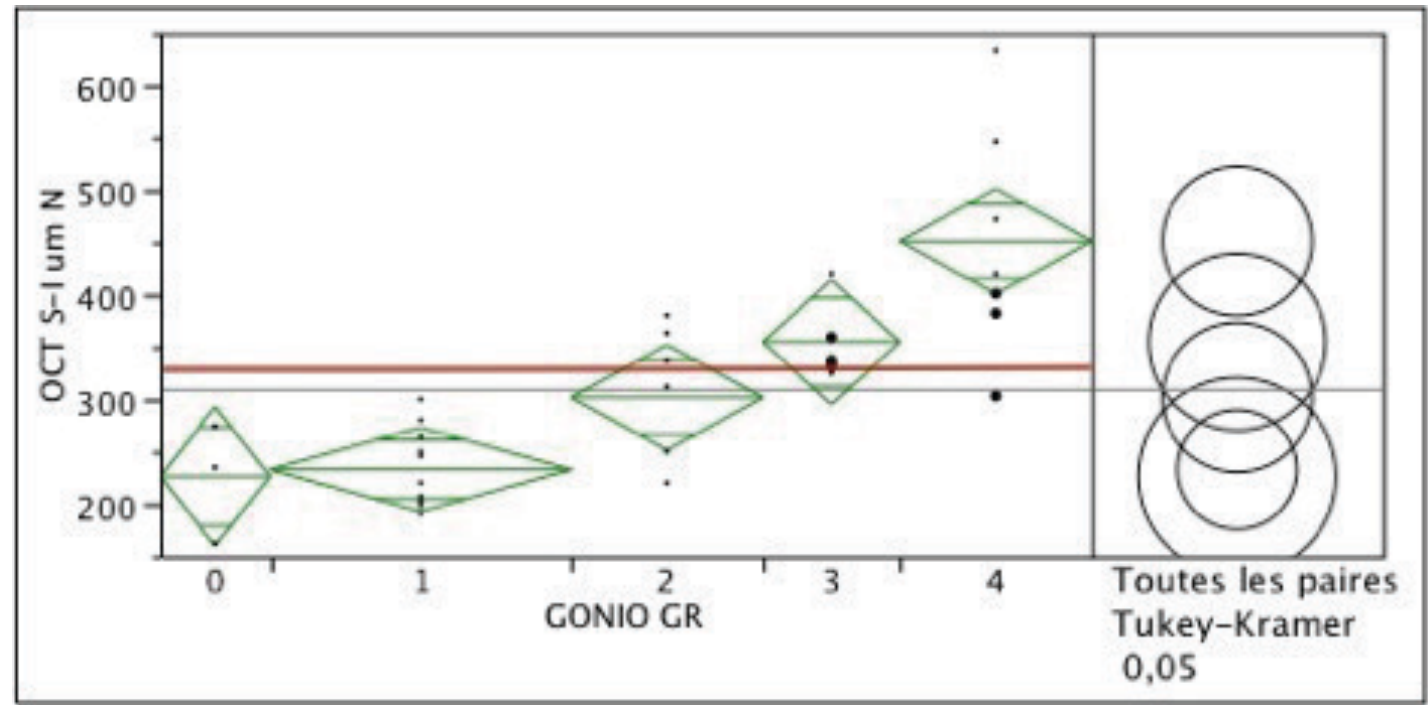

L'analyse du CCI a montré que les calculs du CCI inter-observateur avaient une excellente reproductibilité (0,94 et $0,93, p<0,001$ pour les quadrants nasal et temporal respectivement). En utilisant la même méthode, l'analyse a également révélé une excellente reproductibilité intra-observateur $(0,98$ et $0,99, p<0,001$ pour les quadrants nasal et temporal respectivement).

DISCUSSION

Cette étude visait à concevoir un nouveau paramètre simple pour quantifier le risque de fermeture de l'angle, en utilisant la ligne de Schwalbe identifiée sur l'image par TCO du segment antérieur. Éliminant la nécessité de transférer des images sur certains des instruments de TCO disponibles, d'utiliser des logarithmes mathématiques complexes ou de manipuler plusieurs points de compas sur des images acquises, nos résultats ont démontré une excellente corrélation entre nos mesures S-I et le classement par gonioscopie standard. Cette mesure quantitative simple peut être utilisée pour faire un dépistage rapidement chez les patients chez lesquels on soupçonne une fermeture de l'angle tout en éliminant la nécessité d'anesthésier par voie topique et de créer un contact avec la cornée du patient. De plus, cette technique nécessite moins de manipulations de l'opérateur pour effectuer des calculs par rapport à des méthodes comme les mesures TISA et AOD et élimine le besoin de transfert d'images.

Avec l'avènement de l'imagerie haute résolution de l'angle de la chambre antérieure, la visualisation de la ligne de Schwalbe est désormais plus facile ; elle a été identifiée dans $94 \%$ des images analysables, un résultat semblable à ceux des études précédentes ${ }^{10,15}$. D'autres chercheurs ont utilisé l'éperon scléral ou le réseau trabéculaire comme repères anatomiques pour la mesure de leurs paramètres ${ }^{16-17}$. Une limitation majeure de l'utilisation de ces repères est qu'ils ne sont identifiables que sur 70 à $80 \%$ des images TCO-HD. Ainsi, nos résultats ont montré que notre paramètre utilisant la ligne de Schwalbe comme point de repère anatomique peut être plus approprié que la TCO pour évaluer les angles irido-cornéens.

La figure 3 est une illustration graphique des résultats du test DSH de Tukey-Kramer pour le quadrant nasal. Les résultats sont semblables à ceux obtenus pour le quadrant temporal (résultats non présentés). Les cercles de comparaison des valeurs S-I correspondant aux grades 3 et 4 établis par gonioscopie et des valeurs S-I correspondant aux angles à risque de fermeture d'après la gonioscopie montrent une différence statistiquement significative. La ligne rouge affiche la valeur seuil S-I $(330 \mathrm{~mm})$ entre les angles ouverts et ceux à risque de fermeture, telle que définie par l'analyse ROC et l'identification subséquente de l'indice de Younen (tableau non présenté).

L'une des principales limites de cette étude est le manque d'acquisition d'images TCO-HD pour les quadrants supérieur et inférieur, en raison de difficultés techniques (paupières et besoin d'un speculum). Il est possible que le 
schéma de visibilité des repères anatomiques dans ces quadrants soit différent. Deuxièmement, avec l'imagerie TCO-HD, une lampe de fixation externe doit être utilisée pour bien positionner l'œil du sujet et visualiser les quadrants horizontaux. La position de fixation de la lampe de fixation n’a pas encore été normalisée. De plus, tous les sujets de l'étude étaient de race blanche. La fermeture de l'angle étant plus répandue dans d'autres ethnies, comme les Asiatiques, les Afro-Américains et les Inuits, il pourrait être très utile de répéter l'étude sur ces groupes pour mieux caractériser S-I dans différentes populations. Enfin, la taille relativement petite de l'échantillon constitue une autre limite potentielle.

\section{CONCLUSION}

Le glaucome primitif à angle fermé est une maladie qui peut causer la cécité. Les méthodes actuelles de dépistage comprennent la gonioscopie et l'UBM, qui exigent toutes deux le contact de surface avec l'œil et sont difficiles à maitriser. L'imagerie du segment antérieur par TCO-HD est une méthode prometteuse et non invasive, mais son utilisation n'est pas normalisée pour le dépistage des angles à risque de fermeture. Les résultats obtenus en utilisant notre nouveau paramètre de mesure, méthode qui peut s'avérer plus simple pour certains utilisateurs, montrent que S-I pourrait être utile pour quantifier rapidement et facilement le risque de fermeture de l'angle. Une valeur seuil entre l'angle irido-cornéen ouvert et fermé a également été fournie provisoirement $(330 \mathrm{~mm})$. D'autres études devront être menées pour répondre aux limites susmentionnées.

\section{Nom et adresse de courriel de l'auteur correspondant :}

Dan Samaha, dan.samaha@umontreal.ca, (514)880-3266

Aucune subvention ou compensation financière n’a été reçue pour la rédaction de cet article.

\section{RÉFÉRENCES}

1. Quigley HA, Broman AT. The number of people with glaucoma worldwide in 2010 and 2020. Br J Ophthalmol 2006; 90: 262-7.

2. Patel K, Patel S. Angle-closure glaucoma. DM 2014; 60: 254-62.

3. Foster PJ, Devereux JG, Alsbirk PH, et al. Detection of gonioscopically occludable angles and primary angle closure glaucoma by estimation of limbal chamber depth in Asians: modified grading scheme. Br J Ophthalmol 2000; 84: 186-92.

4. Friedman DS, He M. Anterior chamber angle assessment techniques. Surv Ophthalmol 2008; 53: 250-73.

5. Schirmer KE. Gonioscopy and artefacts. Br J Ophthalmo 1967; 51: 50-3.

6. Huang D, Swanson EA, Lin CP, et al. Optical coherence tomography. Science 1991; 254: 1178-81.

7. Radhakrishnan S, Rollins AM, Roth JE, et al. Real-time optical coherence tomography of the anterior segment at $1310 \mathrm{~nm}$. Arch ophthalmol 2001; 119: 1179-85.

8. Leung CK, Li H, Weinreb RN, et al. Anterior chamber angle measurement with anterior segment optical coherence tomography: a comparison between slit lamp OCT and Visante OCT. Invest Ophthalmol Vis Sci 2008; 49: 3469-74.

9. Muller M, Dahmen G, Porksen E, et al. Anterior chamber angle measurement with optical coherence tomography: intraobserver and interobserver variability. J Cataract Refract Surg 2006; 32: 1803-8.

10. Qin B, Francis BA, Li Y, et al. Anterior chamber angle measurement using schwalbe's line with high resolution fourier-domain optical coherence tomography. J Glaucoma 2013; 22: 10.1097/ IJG.0b013e318264b921

11. Dinc UA, Oncel B, Gorgun E, et al. Assessment of anterior chamber angle using Visante OCT, slit-lamp OCT, and Pentacam. Eur J ophthalmol 2010; 20: 531-7.
12. Doors M, Cruysberg LP, Berendschot TT, et al. Comparison of central corneal thickness and anterior chamber depth measurements using three imaging technologies in normal eyes and after phakic intraocular lens implantation. Graefes Arch Clin Exp Ophthalmol 2009; 247: 1139-46.

13. Lange S, Haigis W, Grein HJ, et al. Comparison of different optical techniques for determination of the dimensions of anterior ocular segment. Klin Monbl Augenheilkd 2009; 226: 485-90.

14. Rossi GC, Scudeller L, Delfino A, et al. Pentacam sensitivity and specificity in detecting occludable angles. Eur J ophthalmol 2012; 22: 701-8.

15. Wong HT, Lim MC, Sakata LM, et al. High-definition optical coherence tomography imaging of the iridocorneal angle of the eye. Arch ophthalmol 2009; 127: 256-60.

16. Liu S, Li H, Dorairaj S, et al. Assessment of scleral spur visibility with anterior segment optical coherence tomography. J Glaucoma 2010; 19: 132-5.

17. Sakata LM, Lavanya R, Friedman DS, et al. Assessment of the scleral spur in anterior segment optical coherence tomography images. Arch ophthalmol 2008; 126: 181-5.

18. Antoniazzi E, Pezzotta S, Delfino A, et al. Anterior chamber measurements taken with Pentacam: an objective tool in laser iridotomy. Eur J ophthalmol 2010; 20: 517-22.

19. Kurita N, Mayama C, Tomidokoro A, et al. Potential of the pentacam in screening for primary angle closure and primary angle closure suspect. J Glaucoma 2009; 18: 506-12. 